\section{CANADIAN ENTOMOLOGIST}

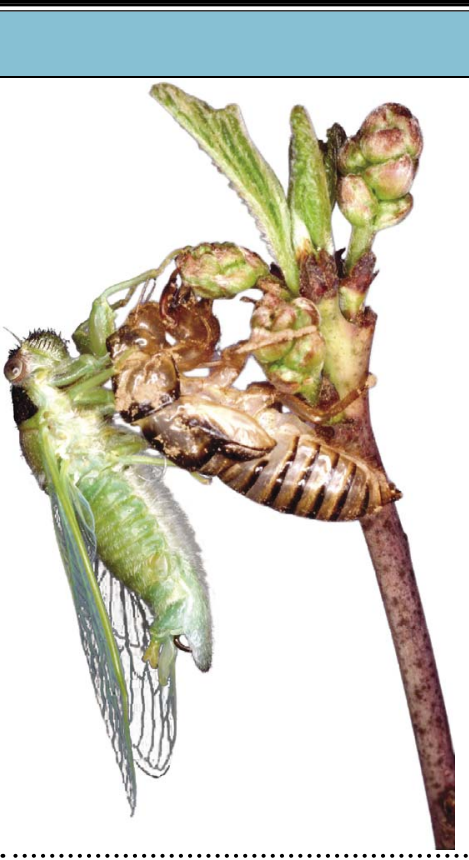

VOLUME 146

NUMBER 5

OCTOBER 2014

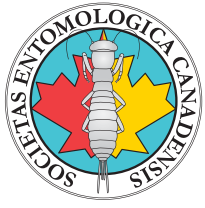

Published Bi-monthly by

Cambridge University Press

www.cambridge.org

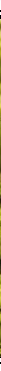




\section{The Canadian Entomologist}

Manuscripts. The Canadian Entomologist accepts manuscript submissions through the ScholarOne Manuscripts system at http:// mc.manuscriptcentral.com/tce. For questions concerning "General Information and Instructions to Authors," please contact the Editor Christopher Buddle at chris.buddle@mcgill.ca, 514-398-8026.

Publishing, Subscription, and Advertising Offices. Cambridge University Press, 32 Avenue of the Americas, New York, NY 100132473, U.S.A.; or Cambridge University Press, The Edinburgh Building, Shaftesbury Road, Cambridge CB2 8RU, England.

The Canadian Entomologist (ISSN 0008-347X) is published bimonthly by Cambridge University Press, 32 Avenue of the Americas, New York, NY 10013-2473; Cambridge University Press, The Edinburgh Building, Shaftesbury Road, Cambridge CB2 8RU, UK. Annual subscription rates for Volume 146, 2014: Institutions print and online $£ 316 / \$ 506$; institutions online only $£ 213 / \$ 340$; single part $£ 57.93 / \$ 92.76$, payable in advance or on receipt of invoice; institutional check or credit card only. The Canadian Entomologist and all other Cambridge Journals can be found at http://journals.cambridge.org/

(C) Entomological Society of Canada 2014. All rights reserved. No part of this publication may be reproduced, in any form or by any means, electronic, photocopying, or otherwise, without permission in writing from Cambridge University Press. Policies, request forms, and contacts are available at: http:www.cambridge.org/rights/permissions/permission.htm

The Entomological Society of Canada grants permission to individuals to make single copies of articles for personal use. Short excerpts from articles in the journal may be reproduced for any purpose that respects the moral rights of authors, provided that the source is fully acknowledged.

Although the Entomological Society of Canada holds the copyrights to The Canadian Entomologist, it does not hold the copyrights to the contents of any papers published prior to 2002. Therefore, authorization to reproduce items for other than personal use, as stated above, for material published before 2002 requires the consent of the authors or their descendants. Authorization to reproduce material published since 2002 can be obtained by contacting the Chair of the Publications Committee, Tom Lowery, at Tom.Lowery@AGR.GC.CA.

The Entomological Society of Canada acknowledges the financial support of the Government of Canada, through the Publications Assistance Program, toward mailing costs in Canada.

http://esc-sec.org/

Entomological Society of Canada, 393 Winston Avenue, Ottawa, Ontario, Canada K2A 1Y8

Publications Mail Registration No. 09572

Publications Mail Agreement No. 40033986

Date of issue: October 2014

Permission to copy (for users in the U.S.A.) is available from Copyright Clearance Center, http://www.copyright.com, email: info@ copyright.com.

Periodicals postage paid at New York, NY, and additional mailing offices.

Postmaster: Send address changes in the U.S.A., Canada, and Mexico to: The Canadian Entomologist, Journals Dept., Cambridge University Press, 100 Brook Hill Drive, West Nyack, NY 10994-2133, U.S.A. Send address changes elsewhere to: The Canadian Entomologist, Journals Dept., Cambridge University Press, The Edinburgh Building, Shaftesbury Road, Cambridge CB2 8RU, England.

Manuscrits. The Canadian Entomologist accepte les soumissions de manuscrits par le système ScholarOne Manuscripts sur http:// mc.manuscriptcentral.com/tce. Pour des questions concernant les informations générales et les instructions aux auteurs veuillez contacter le rédacteur en chef, Christopher Buddle par courriel chris.buddle@mcgill.ca ou téléphone 514-398-8026.

Bureaux de publication, abonnements et publicité . Cambridge University Press, 32 Avenue of the Americas, New York, NY 100132473, É.-U. ; ou Cambridge University Press, The Edinburgh Building, Shaftesbury Road, Cambridge CB2 8RU, Angleterre.

The Canadian Entomologist (ISSN 0008-347X) est publié tous les deux mois par Cambridge University Press, 32 Avenue of the Americas, New York, NY 10013-2473 ; Cambridge University Press, The Edinburgh Building, Shaftesbury Road, Cambridge CB2 8RU, UK. Abonnement annuel pour le volume 146, 2014 : version papier et en ligne pour les institutions $316 £ / 506 \$$; version en ligne seulement pour les institutions $213 £ / 340 \$$; pièce unique $57.93 £ / 92.76 \$$, payables à l'avance ou sur réception de la facture ; chèque institutionnel ou carte de crédit seulement. The Canadian Entomologist et les autres revues de Cambridge se trouvent sur http://journals. cambridge.org/

(C) La Société d'entomologie du Canada 2014. Tous droits réservés. Aucune partie de cette publication ne peut être reproduite, sous quelque forme ou par quelque moyen que ce soit, électronique, photocopie, ou autre, sans l'autorisation écrite de Cambridge University Press. Les politiques, formulaires de demande, et les contacts sont disponibles sur : http://www.cambridge.org/rights/permissions/ permission.htm

La Société d'entomologie du Canada donne la permission à des individus de faire des copies uniques des articles pour usage personnel. De courtes sections des articles peuvent être reproduites pour tout usage qui respecte le droit moral des auteurs, en autant que la source est pleinement reconnue.

Bien que la Société d'entomologie du Canada détienne les droits d'auteur de la revue The Canadian Entomologist, elle ne possède pas ces droits pour tout article publié avant 2002. Par conséquent, l'autorisation pour reproduire du matériel publié avant 2002 pour toutes autres fins que personnelles, tel que cité précédemment, requière le consentement de l'auteur ou de ses descendants. Vous pouvez obtenir l'autorisation de reproduire du matériel publié depuis 2002 en contactant le président du Comité des publications, Tom Lowery, àTom.Lowery@AGR.GC.CA.

La Société d'entomologie du Canada reconnaît l'apport financier du programme d'assistance publique du Gouvernement du Canada pour son aide aux frais de postes au Canada.

http://esc-sec.org/

La Société d'entomologie du Canada, 393, avenue Winston, Ottawa, Ontario, Canada K2A 1Y8

Poste-publications enregistrement $\mathrm{n}^{\circ} 09572$

Convention du service poste-publication $\mathrm{n}^{\circ} 40033986$

Date de publication: octobre 2014

La permission de reproduire (pour les utilisateurs aux États-Unis) est disponible à partir du Copyright Clearance Center, http://www. copyright.com, courriel : info@copyright.com.

Port payé à New York, NY, et dans les bureaux de poste supplémentaires.

Maître de poste : Envoyer les changements d'adresse aux États-Unis, au Canada et au

Mexique à : The Canadian Entomologist, Journals Dept, Cambridge University Press, 100 Brook Hill Drive, West Nyack, NY 109942133, États-Unis. Envoyer tout changement d'adresse ailleurs dans le monde à : The Canadian Entomologist, Journals Dept, Cambridge University Press, The Edinburgh Building, Shaftesbury Road, Cambridge CB2 8RU, Angleterre. 


\section{The Canadian Entomologist}

Volume 146

October 2014

Number 5

\section{Systematics \& Morphology}

A. Loghmani, H. Hajiqanbar, A.A. Talebi-An illustrated key to world species of the mite family Trochometridiidae (Acari: Prostigmata), with description of a new species and new insect host

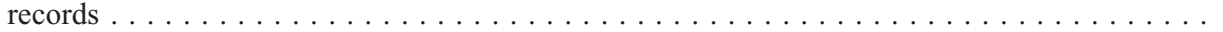

O. Lonsdale - Redefinition and synonymy of genera in the Ophiomyia genus group, with the description of Euhexomyza new genus (Diptera: Agromyzidae) . . . . . . . . . . . .

Behaviour \& Ecology

M. Hrabar, A. Danci, S. McCann, P.W. Schaefer, G. Gries-New findings on life history traits

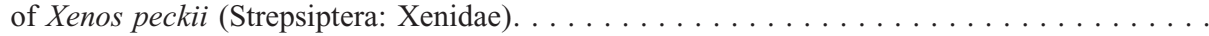

V. Hock, G. Chouinard, É. Lucas, D. Cormier, T. Leskey, S. Wright, A. Zhang,

A. Pichette-Establishing abiotic and biotic factors necessary for reliable male pheromone production and attraction to pheromones by female plum curculios Conotrachelus nenuphar

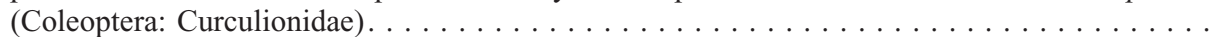

K.M. Ablard, K. Simonetto, L.K. Weir, B.J. Crespi, P.W. Schaefer, G. Gries-First-male sperm precedence and precopulatory and postcopulatory rituals in the parasitoid wasp Ooencyrtus kuvanae (Hymenoptera: Encyrtidae) . . . . . . . . . . . . . . . . . .

\section{Biodiversity \& Evolution - NOTE}

V.A.D. Hervet, H. Murillo, J.L. Fernández-Triana, M.R. Shaw, R.A. Laird,

K.D. Floate-First report of Cotesia vanessae (Hymenoptera: Braconidae) in North America

Insect Management

J.M. Renkema, G.C. Cutler, D. Blanchard, A. Hammermeister-Using ground beetles (Coleoptera: Carabidae) to control slugs (Gastropoda: Pulmonata) in salad greens in the laboratory and greenhouse . . . . . . . . . . . . . . . . . . . .

Insect Management - NOTE

T.D. Gariepy, H. Fraser, C.D. Scott-Dupree - Brown marmorated stink bug (Hemiptera: Pentatomidae) in Canada: recent establishment, occurrence, and pest status in southern Ontario 


\section{The Canadian Entomologist}

\section{Editor-in-Chief/Directeur scientifique en chef}

Christopher M. Buddle

Sainte-Anne-de-Bellevue, Québec

\section{Co-Editor-in-Chief/Co-Directeur scientifique en chef}

Kevin Floate

Lethbridge, Alberta

\section{Editorial Assistant/Adjoint à la rédaction}

Andrew B.T. Smith

Ottawa, Ontario

\section{Subject Editors/Rédacteurs thématiques}

\begin{tabular}{|c|c|c|}
\hline Gilles Boiteau & Dylan Parry & Cory Sheffield \\
\hline Fredericton, New Brunswick & Syracuse, New York & Regina, Saskatchewan \\
\hline Patrice Bouchard & Heather Proctor & Bradley Sinclair \\
\hline Ottawa, Ontario & Edmonton, Alberta & Ottawa, Ontario \\
\hline Hervé Colinet & Deepa Pureswaran & Brent Sinclair \\
\hline Rennes, France & Sainte-Foy, Québec & London, Ontario \\
\hline Robert Johns & Kateryn Rochon & Keith Summerville \\
\hline Fredericton, New Brunswick & Winnipeg, Manitoba & Des Moines, Iowa \\
\hline Staffan Lindgren & Chris Schmidt & Jianghua Sun \\
\hline Prince George, British Columbia & Ottawa, Ontario & Beijing, China \\
\hline Véronique Martel & Justin Schmidt & Jon Sweeney \\
\hline Ville de Québec, Québec & Tucson, Arizona & Fredericton, New Brunswick \\
\hline David McCorquodale & Michael J. Sharkey & \\
\hline Sydney, Nova Scotia & Lexington, Kentucky & \\
\hline
\end{tabular}


